www.periodicos.unimontes.br/index.php/caminhosdahistoria

\title{
INTERCÂMBIO CIENTÍFICO: AS RELAÇÕES BRASIL - ALEMANHA ATRAVÉS DAS LENTES DO COLPOSCÓPIO EM MEADOS DO SÉCULO XX
}

Resumo: O objetivo deste texto é discutir a aproximação das ciências brasileira e alemã, através da introdução e difusão da colposcopia no Brasil. O colposcópio, desenvolvido pelo médico alemão Hans Hinselmann, nos anos 1920, facilitava o diagnóstico das patologias ginecológicas, tornando-se referência para detecção do câncer do colo do útero na primeira metade do século XX. No Brasil, houve uma particularidade na organização de ações para diagnóstico do câncer do colo do útero, que refletiu na elaboração de um modelo próprio de atendimento. Esta particularidade pode ser explicada por uma aproximação entre as ciências alemã e brasileira nos anos 1930 e pelas próprias diretrizes da saúde nacional no período. A partir desses pontos, será analisado o processo de criação e divulgação da técnica, o uso do colposcópio no país e os diálogos entre os médicos brasileiros e Hans Hinselmann, na afirmação da ferramenta para o controle da doença no território brasileiro em meados do século XX.

Palavras-chave: Intercâmbio científico; Colposcopia; História das Ciências; Câncer do Colo do Útero; Ginecologia.

Abstract: The purpose of this text is to discuss the approximation of the Brazilian and German sciences, through the introduction and diffusion of colposcopy in Brazil. The colposcope, developed by the German physician Hans Hinselmann in the 1920s, facilitated the diagnosis of gynecological pathologies, becoming a reference for the detection of cervical cancer in the first half of the 20th century. In Brazil, there was a particularity in the organization of actions for the diagnosis of cervical cancer, which reflected in the development of its own model of care. This particularity can be explained by an approximation between the German and Brazilian sciences in the 1930s and by the national health guidelines themselves in the period. From these points, the process of creating and disseminating the technique, the use of the colposcope in the country and the dialogues between Brazilian doctors and Hans Hinselmann will be analyzed, in the affirmation of the tool for the control of the disease in the Brazilian territory in the middle of the 20th century.

Keywords: Scientific exchange; Colposcopy; History of Science; Cervical Cancer; Gynecology.

O colposcópio é uma ferramenta de visualização do colo uterino, utilizada para detecção de lesões no órgão e diagnóstico de diferentes doenças ginecológicas. O aparelho foi

\footnotetext{
${ }^{1}$ Doutora em História das Ciências; Pós-Doutorado pela Casa de Oswaldo Cruz/FIOCRUZ. Professora do Departamento de História da Universidade Federal de Viçosa e do Mestrado em Patrimônio Cultural, Paisagens e Cidadania. E-mail: vanessalana@ufv.br. https://orcid.org/0000-0002-1950-1206.
} 
desenvolvido pelo médico alemão Hans Hinselmann² na década de 1920 e começou a ser utilizado pela medicina brasileira nos anos 1930. A ferramenta teve impacto efetivo na realização da detecção precoce do câncer do colo do útero. Este diagnóstico era visto pela medicina como a principal estratégia para controle da doença, em decorrência dos limites em tratar e curar pacientes em estágios avançados de evolução do tumor.

A introdução e difusão de novas tecnologias variam de acordo com questões culturais, econômicas e políticas dos contextos nos quais se inserem. Os próprios usos e consequências são moldados a partir das estruturas sociais, interesses profissionais e governamentais (Timmermann and Anderson, 2006). O colposcópio, neste sentido, teve diferentes inserções nos cenários médicos alemão e de outros países. Em linhas gerais, para além das nações de língua alemã, como países da Europa ocidental e os EUA, o colposcópio não fui utilizado como primeira ferramenta no diagnóstico do câncer do colo do útero. Nestes locais, a citologia $^{3}$, desenvolvida pelo médico grego Papanicolaou, foi utilizada como ferramenta principal em relação à doença, sendo a colposcopia utilizada como método auxiliar. No entanto, em alguns países latino-americanos, como o Brasil, a inserção da tecnologia alemã ocorreu de forma quase simultânea ao seu desenvolvimento, sendo utilizada como principal estratégia para controle do câncer.

No Brasil, houve uma particularidade na organização de ações para diagnóstico do câncer do colo do útero, que refletiu na elaboração de um modelo próprio de atendimento, identificado pela literatura como "modelo triplo" (Lana, 2012; Teixeira e Lowy, 2011). Esta particularidade pode ser explicada por uma aproximação entre as ciências alemã e brasileira nos anos 1930 e pelas próprias diretrizes da saúde nacional no período. Posto isso, discutiremos nesse texto a aproximação das ciências brasileira e alemã, através da introdução e difusão da colposcopia no Brasil. Para tal, percorreremos o processo de criação e divulgação da técnica, o uso do colposcópio no país e os diálogos entre os médicos brasileiros e Hans Hinselmann, na afirmação da ferramenta para o controle da doença em meados do século XX.

\footnotetext{
${ }^{2}$ Hans Hinselmann nasceu em seis de agosto de 1884 na cidade alemã de Neumunster. Cursou medicina em Heidelberg e Kiel, graduando-se nesta última em 1908. No ano de 1909 iniciou sua especialização em ginecologia em Jena, trasferindo-se para Geissen em 1910 e concluindo a especialidade em Bonn, em 1911 (Powel, 2004).

${ }^{3}$ Em 1917, Papanicolaou, a partir da análise das células presentes no esfregaço vaginal, observou a existência de fases rítmicas do ciclo sexual. Da continuidade de seus estudos, ao final dos anos 1920, verificou-se a possibilidade do reconhecimento de células cancerosas no conteúdo vaginal pela análise microscópica das células obtidas por esfregaço (Lowy, 2010).
} 


\section{A colposcopia na Alemanha}

O colposcópio foi desenvolvido em 1924. Naquele período, Hans Hinselmann trabalhava na Clínica Ginecológica da Universidade de Bonn e recebeu um convite do então diretor, Professor Von Franqué, para a elaboração conjunta de um capítulo sobre o câncer do colo do útero para uma nova edição do manual de ginecologia alemão organizado por Veit Stoeckel, o "Handbuch der Gynäkologie"4 . Segundo o próprio Hinselmann, a principal ideia que motivou o desenvolvimento da técnica era de que se era possível o reconhecimento de uma lesão de cerca de $1 \mathrm{~mm}$ a olhos nus, seria também possível o reconhecimento na extensão de $0,1 \mathrm{~mm}$ com o emprego de um material óptico que permitisse um aumento de 10 vezes na área visualizada (Hinselmann, 1952).

Hinselmann acreditava que o tumor cancerígeno não poderia evoluir de células normais. Havia a necessidade de estados de transição, com alterações na estrutura e reprodução celular que, no caso do câncer do colo do útero seriam as "zonas matrizes" (Hinselmann, 1952). Essas lesões precursoras, que indicavam a possibilidade de desenvolvimento de um estado cancerígeno, seriam reconhecidas pelo colposcópio, o que permitiria o estudo de alterações microscópicas no órgão.

O contexto político de criação e difusão da colposcopia na Alemanha foi marcado pela ascensão do regime nazista liderado por Adolf Hitler. No campo da saúde, o governo nazista empreendeu uma política de saúde pública que combinava estratégias de pesquisa e regulamentação para doenças ocupacionais, ambientais e ligadas ao estilo de vida. A estruturação dos regimes de extrema direita tinha relação com a organização destas políticas, na formação e manutenção de "homens saudáveis" e da pureza da raça. Das doenças em foco, destacava-se o câncer, considerado nos anos 1930 "inimigo número um do Estado" (Proctor, 1999).

Muitos cientistas viram no regime nazista a possibilidade de garantir status pessoal e financiamento para suas pesquisas. Naquele período, médicos locais foram responsáveis por um grande número de esterilizações involuntárias, eutanásias e experimentos em campos de concentração. Num dos maiores e mais conhecidos campos de concentração nazista, em Auschwitz, um dos experimentos realizados foi o exame de lesões cancerígenas do colo uterino, com utilização do colposcópio. Entre os ginecologistas participantes, estava o nome

A atividade clínica de Hinselmann foi interrompida durante a Primeira Guerra Mundial. Ao final do conflito, com a derrota alemã e as retaliações impostas ao país no Tratado de Versalhes em 1918, Hinselmann voltou a exercer a profissão médica e, em 1921 recebeu o título de professor de ginecologia na Universidade de Bonn (Powell, 2004).

${ }^{4}$ Viet-Stockel. Handbuch der Gynakologie Bd611 854, Bergmann, Munchen, 1930. 
de Hans Hinselmann (Halioua, 2010). Na década de 1930, Adolf Hitler lançou um projeto em grande escala de prevenção do câncer. Com ênfase no público feminino, o projeto fazia uso da propaganda para convencer as mulheres a se submeterem a exames periódicos para diagnóstico e tratamento da doença. O projeto aconteceu concomitante à divulgação da colposcopia como método de controle do câncer do colo do útero, pelo diagnóstico precoce do tumor e extração do mesmo (Idem).

A difusão da técnica na Alemanha, no entanto, ocorreu também cercada por críticas da comunidade médica, principalmente quanto aos custos de aquisição e manutenção do aparelho (Hinselmann, 1952). Tais críticas, por sua vez, contribuíram para estudos de desenvolvimento técnico da ferramenta, como na segurança no emprego da luz e maior precisão na detecção de lesões. No entanto, na comunidade médica alemã, a utilidade e importância do colposcópio para o diagnóstico do câncer do colo do útero não foram alvo de questionamentos.

Todavia, a difusão da colposcopia para além do território alemão ocorreu de forma tímida em muitos países, principalmente da Europa e nos EUA. Dentre os fatores explicativos que podem ser apontados, estão as modificações nas relações diplomáticas no período entre guerras, que geraram um isolamento político da Alemanha nazista e a questão da língua. Sobre o último ponto, como a grande maioria dos escritos sobre colposcopia era de autoria do próprio Hinselmann, a maior parte das publicações estava em alemão, e poucas traduções ou trabalhos originais em inglês, por exemplo. A parca circulação de publicações limitou o conhecimento e utilização da ferramenta, uma vez que o manuseio do aparelho e interpretação das imagens estavam diretamente relacionados ao conhecimento tanto da técnica quanto das discussões acerca da mesma.

Por outro lado, a introdução e difusão da colposcopia em países da América do Sul como Argentina, Brasil e Chile ocorreu quase que instantaneamente à criação e difusão da ferramenta na Alemanha. Esta especificidade em relação ao uso da colposcopia como primeira ferramenta de diagnóstico para o câncer do colo do útero foi designada pela literatura como "exceção latino-americana" (Eraso, 2010). Esta "exceção" configurou-se na utilização da colposcopia como primeira ferramenta de diagnóstico, de forma diferente ao verificado em países europeus e nos EUA, nos quais a citologia foi o principal método nas campanhas de prevenção da doença.

A aproximação da ciência na América Latina com a ciência alemã na primeira metade do século XIX é um dos indicativos que explica a introdução e difusão da colposcopia nos países latino-americanos de forma concomitante ao desenvolvimento da ferramenta em terras alemãs. O desenvolvimento da colposcopia como método de diagnóstico em países como o 
Brasil reflete um emaranhado de interesses políticos e iniciativas locais, atendendo às configurações de saúde de cada país (Eraso, 2010; Teixeira and Lowy, 2011).

A literatura que se dedicou à questão da colposcopia em meados do século XX destacou os escritos latino-americanos, especialmente os brasileiros, como referências no estudo da ferramenta (Scheffey, Bolten, and Warren, 1955). Segundo essa literatura, além de traduções do alemão, nos periódicos médicos desses países era possível encontrar um quantitativo significativo de publicações sobre o tema e sobre experiências locais na utilização da técnica, principalmente a partir dos anos 1940.

Os anos que se seguiram ao final da Primeira Guerra Mundial foram marcados por uma reaproximação científica da Alemanha com países latino-americanos. Além da questão científica, os germânicos viam na região um potencial para aquisição de matérias-primas e absorção do excedente industrial (Sá, 2009). A partir da década de 1920, as relações científicas foram tecidas com a divulgação de periódicos em espanhol e português, que destacavam os feitos da ciência alemã, financiamento para pesquisas, viagens científicas e a criação de sociedades médicas para angariar fundos para tais atividades e agregar os profissionais envolvidos (Sá e Silva, 2007).

Exemplos desta interlocução eram as revistas médicas como a "Revista Médica de Hamburgo" (posteriormente "Revista Médica Germano-Ibero-Americana), que divulgava às comunidades médicas espanhola e latino-americanas os experimentos realizados pela medicina alemã, principalmente no tocante a produtos da indústria farmacêutica e questões relacionadas à terapêutica (Sá e Silva, 2007). A medicina, neste sentido, atuava como veículo de divulgação científica e cultural germânica, atrelada aos setores da política e economia.

Em relação à ginecologia e obstetrícia, França e Alemanha eram, desde o fím do século XIX, as principais referências em escolas médicas no mundo ocidental. A partir da década de 1930, mesmo com grande influência exercida pela medicina francesa na educação médica latino-americana, observou-se uma forte aproximação com a medicina alemã, principalmente no campo da saúde da mulher. A partir de então, observou-se uma maior interlocução científica entre estes espaços, o que funcionou como um importante veículo na transferência de ideias e tecnologias médicas (Eraso, 2010).

\section{A colposcopia no Brasil}

O colposcópio foi introduzido no Brasil pelo ginecologista carioca Arnaldo de Moraes. Como catedrático de Clínica Ginecológica na Faculdade Fluminense de Medicina, na década 
de 1930, o médico tomou conhecimento da técnica desenvolvida por Hinselmann a partir das leituras do Tratado de Ginecologia de Stoeckel. Foi ainda nos anos 1930 que Moraes adquiriu os primeiros aparelhos: um para o ambulatório de sua clínica na Faculdade, em 1934 e, logo após, um segundo para seu consultório particular. Em 1935 o médico prestou concurso de catedrático de Clínica Ginecológica na Faculdade de Medicina da Universidade do Brasil. Na prova prática do concurso, utilizou o colposcópio para examinar uma paciente, identificando lesões no colo uterino (Rieper, 1950). Logo após assumir a cátedra de Ginecologia do referido concurso, em 1937, adquiriu novo colposcópio para utilização na Faculdade. Moraes exaltava a importância da ferramenta justificando que somente o exame ginecológico pela visão direta a olho nu, com aumentos no ângulo de visão e luz apropriada, poderia identificar lesões suspeitas a serem ou não confirmadas pela biópsia (Moraes, 1952).

O uso do colposcópio para diagnóstico do câncer do colo do útero na Clínica Ginecológica da Faculdade de Medicina ganhou fôlego após 1940, quando o médico João Paulo Rieper, discípulo de Hinselmann, com o qual fizera um curso na cidade alemã de Hamburgo, iniciou seus trabalhos no Serviço dirigido por Arnaldo de Moraes, em funcionamento no Hospital Estácio de Sá no Rio de Janeiro. Rieper esteve na Alemanha na década de 1930, período no qual se aproximou da ciência alemã e foi primeiro secretário da Academia Médica Germano Ibero-Americana, na qual auxiliava nas funções de recepção de estudantes e professores latino-americanos no país (Lana, 2016).

A Academia Médica Germano Ibero-Americana foi criada em 1935, com o objetivo de promover o intercâmbio acadêmico entre a Alemanha e os médicos latino-americanos. A década de 1930 foi marcada pela formação de uma política cultural Alemanha-Brasil, com esforços de diplomacia cultural que se fizeram presentes principalmente na área médica. Estes esforços ocorreram, por exemplo, na vinda e contratação de professores alemães para universidades brasileiras, como a Universidade de São Paulo, na década de 1930 (Silva, 2013). A própria passagem de Rieper pela Alemanha esteve inserida no contexto de coordenação e incentivo ao intercâmbio científico do país com outras localidades. Esta diplomacia cultural alemã foi impulsionada por um espírito nacionalista, que norteou as relações transnacionais após a Primeira Guerra Mundial (Idem).

Rieper graduou-se em Medicina na cidade de Berlin em 1939, retornando ao Brasil $\operatorname{logo}$ em seguida. Por ocasião da deflagração da deflagração da Segunda Guerra Mundial fora 
impedido de voltar à Alemanha, revalidando seu diploma de médico na Faculdade de Medicina da Universidade do Brasil ${ }^{5}$ e iniciando sua carreira científica na instituição.

A tese de docência apresentada por Rieper na Faculdade: "Sobre o valor prático da colposcopia" era um manual de apresentação da tecnologia e indicação prática de seus benefícios. Rieper defendia que o correto uso do colposcópio somente poderia ser realizado por profissionais preparados diretamente pelo criador do método, Hans Hinselmannn, ou por algum discípulo direto do mesmo. $\mathrm{O}$ estudo da colposcopia apenas por publicações e imagens comentadas não era eficiente para formação de um bom colposcopista (Rieper, 1948). A partir desse preceito, e com impulso da política de formação profissional da Faculdade, o médico brasileiro iniciou a formação de profissionais locais para manuseio do aparelho e instalação de centros de colposcopia no país.

Dentre os profissionais formados por Rieper, com incentivo da política de formação profissional e difusão da colposcopia empreendida pela Clínica dirigida por Arnaldo de Moraes, estava o médico mineiro Alberto Henrique da Rocha. Rocha foi o responsável pela difusão da colposcopia para diagnóstico do câncer do colo do útero no Hospital de Ginecologia da Faculdade de Medicina da Universidade de Minas Gerais dirigido pelo médico e político mineiro Clóvis Salgado. O primeiro colposcópio em Minas Gerais foi adquirido por Clóvis Salgado em 1934, e depois transferido para a faculdade (Lana, 2012).

Alberto Henrique da Rocha iniciou seus trabalhos como assistente no Hospital de Ginecologia em 1941. Desde o início de suas atividades, o médico trabalhou com o exame colposcópico, num modelo de atendimento que defendia a realização do exame em todas as mulheres que buscassem o serviço. Em 1947, para assumir uma cátedra de ensino na Universidade, Rocha publicou sua tese de livre docência com o título: "Contribuição da Colposcopia ao Diagnóstico Ginecológico” (Rocha, 1955).

Os ambulatórios das faculdades de medicina do Rio de Janeiro e de Minas foram os principais espaços de introdução e posterior difusão da colposcopia como ferramenta de diagnóstico do câncer do colo do útero no Brasil. No processo de difusão de uma nova tecnologia médica, as questões culturais exercem grande impacto, sendo o papel das instituições e atores decisivo neste processo (Blume, 1992). Este processo de difusão segue os preceitos do contexto no qual a tecnologia se insere, correspondendo às demandas e organização do sistema de saúde local. Como no Brasil o atendimento à doença tinha nos consultórios de ginecologia e ambulatórios das faculdades de medicina seu principal aporte,

\footnotetext{
5 Documentação da Academia Médica Germano-Ibero-Americana, depositada no Geheimes Staatsarchiv
} Preussischer Kulturbesitz. 
foi nestes espaços que a tecnologia ganhou espaço e visibilidade. No caso do colposcópio, este atendia à proposta de ação sobre a doença, baseada no exame sistemático de todas as mulheres que buscassem atendimento, como ações locais, e sem uma política de exames em um maior número de paciente, na configuração de uma política de controle da enfermidade.

\section{Hinselmann no Brasil}

A aproximação das ciências brasileira e alemã, no tocante à difusão e afirmação da colposcopia como tecnologia de diagnóstico do câncer do colo do útero teve impulso com as viagens realizadas por Hans Hinselmann no Brasil, para divulgação da ferramenta e formação de profissionais. Hinselmann esteve no Brasil por três vezes. A primeira, em 1949, atendeu ao convite de Arnaldo de Moraes para reorganização do Laboratório de Patologia da Faculdade de Medicina, e para realização de palestras sobre a colposcopia e seus avanços no diagnóstico da doença. Por ocasião da visita, a Sociedade Brasileira de Ginecologia realizou em dezembro de 1949 uma sessão extraordinária em homenagem ao médico e para sua recepção. Na sessão, Hinselmann discursou sobre seus trabalhos em colposcopia e enalteceu a importância do intercâmbio estabelecido com o Brasil (Hinselmann, 1950).

O primeiro curso ministrado pelo médico alemão no Brasil aconteceu em dezembro de 1949, no Rio de Janeiro. Hinselmann iniciou o curso descrevendo o percurso de construção do colposcópio, destacando as ideias envolvidas no desenvolvimento da ferramenta. Com objetivo de formação e especialização profissional, as aulas foram ministradas no binômio teoria e prática. A parte teórica era baseada nas explicações do médico sobre o câncer do colo do útero, sobre o colposcópio e os procedimentos para realização do exame de diagnóstico, incluindo questões de nomenclatura e estágio de evolução do tumor. Já a prática esteve organizada a partir de exercícios de análise de desenhos e fotografias para demonstrar os aspectos descritos teoricamente. Na parte final do curso, Hinselmann operou duas pacientes para demonstrar a aplicabilidade da técnica por ele criada (Hinselmam, 1950).

Logo após findar o curso de colposcopia no Rio de Janeiro, Hinselmann seguiu para Belo Horizonte, onde foi recebido por Clóvis Salgado e Alberto Henrique da Rocha. A viagem a Minas não era para cursos de formação, e sim para conhecer a utilização da colposcopia na região e legitimar o emprego da ferramenta nas ações de controle da doença. No segundo dia de sua visita, esteve no ambulatório do Hospital de Ginecologia da Faculdade de Medicina, no qual funcionava o Posto de Combate ao Câncer da Cruz Vermelha. Na ocasião, o médico fez demonstrações sobre a técnica colposcópica e respondeu a dúvidas dos 
profissionais presentes. Ao avaliar os trabalhos ali desenvolvidos, Hinselmann destacou ter ficado impressionado com os dados estatísticos do movimento colposcópico realizado na instituição desde 1940 (Hinselmann, 1950).

Nos anos que antecederam a viagem de Hinselmann à América do Sul, o médico foi diretor do departamento de ginecologia de Hamburg - Altona, na Alemanha, entre os anos de 1940 a 1946. A última operação de Hinselmann na clínica ginecológica em Altona foi em 1946, com a interrupção de uma gravidez e esterilização da paciente. Pouco tempo depois recaiu sobre o médico a acusação de esterilização compulsória de seis mulheres ciganas durante o Terceiro Reich no regime nazista alemão, sem autorização oficial (Halioua, 2010). Com a acusação, o médico foi condenado a três anos de prisão e ao pagamento de multa. Em decorrência do fraco estado de saúde apresentado na prisão, teve a pena reduzida por decisão do júri de generais ingleses, cumprindo dois anos e meio de detenção. Após a prisão, sem autorização para trabalhar em serviços hospitalares, as pesquisas em colposcopia e histopatologia continuaram em seu escritório privado em sua residência, através da prestação de serviços no exame de materiais coletados por terceiros (Powell, 2004). A vinda à América Latina foi a primeira participação de Hinselmann no cenário científico mundial após sair da prisão. O próprio médico considerava sua visita às terras americanas como um aporte à sua carreira, e uma renovação, após as retaliações políticas oriundas da guerra (Hiselmann, 1950).

O processo de afirmação e difusão da tecnologia ficou mais acentuado com a segunda viagem do médico, em 1951, desta vez abrangendo três países sul-americanos: Brasil, Argentina e Uruguai. Em 1955 Hinselmann fez sua terceira e última viagem ao Brasil, na qual recebeu a condecoração do "Doutor Honoris Causa" pela Faculdade de Medicina da Universidade do Brasil, o que representava uma das maiores distinções no meio científico e acadêmico. A viagem foi a última participação do médico no meio científico antes de sua morte em 1959. As condecorações recebidas evidenciavam sua inserção no campo médico brasileiro, os diálogos com a ciência alemã e o fortalecimento da colposcopia como ferramenta de diagnóstico do câncer do colo do útero nas instituições de atendimento à doença no Brasil.

\section{Considerações finais}

No processo de difusão de uma nova tecnologia questões culturais exercem grande influência, a partir principalmente das discussões e ações de instituições e atores tanto na aceitação quanto na incorporação da técnica. Em meados do século XX, a colposcopia 
despertou grande entusiasmo dos médicos brasileiros diante da possibilidade de diminuição da mortalidade por câncer do colo do útero no país, através da perspectiva de detecção precoce.

A aproximação da ciência na América Latina com a ciência alemã na primeira metade do século XIX é um dos indicativos que explica a introdução e difusão da colposcopia nos países latino-americanos, de forma geral a incorporação da colposcopia às ações em saúde ocorreu de forma concomitante ao desenvolvimento da mesma na Alemanha, refletindo tanto um emaranhado de interesses políticos e iniciativas locais, quanto atendendo às configurações de saúde de cada nação. No cenário brasileiro de meados do século XX, a afirmação do colposcópio como tecnologia de diagnóstico do câncer do colo do útero, marcando especificidades nas estratégias de controle da doença em território nacional, foi impulsionada pelo estreitamento das relações entre os médicos brasileiros e alemães, o que foi verificado, por exemplo, nas viagens de Hinselmann ao país e as repercussões tanto para a ciência nacional quanto na própria carreira do médico.

\section{Referências bibliográficas}

BLUME, Stuart. Insight and Industry: on the dynamic of technological change in medicine. Massachusetts Institute of Technology, 1992.

ERASO, Yolanda. "Migrating Tecniques, Multiplying Diagnoses: Tha Contribuition of Argentina and Brazil to Cervical Cancer 'early detection' Policy". História, Ciências, Saúde Manguinhos, Rio de Janeiro, v. 17, supl. 1, julho 2010.

HALIOUA, Bruno. "The Participation of Hans Hinselmann in Medical Experiments at Auschwitz". In: Journal of Lower Genital Tract Disease. Volume 14(1), January 2010, p 1-4. HINSELMAM, Hans. "História da Colposcopia". In: Anais Brasileiros de Ginecologia, 33(2): 65-98, 1952.

HINSELMAM, Hans. "Relatório sobre o curso de colposcopia, de 5 a 17 de dezembro de 1949, no Instituto de Ginecologia, pelo professor Dr. Hans Hinselmann, de Hamburgo". In: Anais Brasileiros de Ginecologia, 29(3): 251-270, 1950.

LANA, Vanessa. Organização da especialidade médica e controle do câncer do colo do útero no Brasil: o Instituto de Ginecologia do Rio de Janeiro em meados do século XX. História, Ciências, Saúde-Manguinhos, v. 23, p. 683-701, 2016.

LANA, Vanessa. Ferramentas, práticas e saberes: a formação de uma rede institucional para a prevenção do câncer do colo do útero no Brasil - 1936-1970. Tese de Doutorado. Casa de Oswaldo Cruz / Fundação Oswaldo Cruz; 2012.

LÖWY, Ilana. Preventive Strikes: Women, Precancer, and Prophylactic Surgery. Baltimore: The Johns Hopkins University Press, 2010.

MORAES, Arnaldo de. "O valor do chamado Dispensário Preventivo na luta contra o câncer genital feminino". In: Anais Brasileiros de Ginecologia, 33(1): 15-28, 1952.

TIMMERMANN, Carsten; ANDERSON, Julie (eds). Devices and designs: medical technologies in historical perspective. Palgrave Macmillan, 2006. 
POWELL, John L. "Biographic Sketch: Powell's Pearls: Hans Hinselmann, MD (1884 1959)". In: Obstetrical and Gynecological Survey. Volume 59, number 10, 2004.

PROCTOR, Robert. Cancer Wars. How Politics Shapes what we Know and don't know about cancer. New York: Basic Books, 1995.

RIEPER, João Paulo. "Evolução da colposcopia no Brasil" - editorial. In: Anais Brasileiros de Ginecologia, 30(6): 462-466, 1950.

ROCHA, Alberto Henrique. "A colposcopia na Clínica Ginecológica da Faculdade de Medicina da Universidade de Minas Gerais (1941-1955)". In: Anais Brasileiros de Ginecologia, 40(3): 219-226, 1955.

SÁ, Magali Romero (et. al.). "Medicina, ciência e poder: as relações entre França, Alemanha e Brasil no período de 1919 a 1942”. In: História, Ciência, Saúde - Manguinhos. v. 16, n.1, p.247-261, jan.-mar. 2009.

SÁ, Magali Romero; SILVA, André Felipe C. "Por entre as páginas do imperialismo germânico na América Latina: a Revista Médica de Hamburgo e a Revista Médica GermanoIbero-Americana (1920-1933)”. In: Simpósio Nacional de História, 24, 2007, São Leopoldo. História e multidisciplinaridade: territórios e deslocamentos. v.1. São Leopoldo: Oikos. p.565. 2007.

SILVA, André Felipe C. "A diplomacia das cátedras: a política cultural externa alemã e o ensino superior paulista - os casos da USP e da Escola Paulista de Medicina (1934-1942)". In: História (São Paulo) v.32, n.1, p. 401-431, jan/jun 2013.

SALGADO, Clóvis; ROCHA, Alberto Henrique; JUNQUEIRA, Moacir. "Do método colposcópico". In: Anais Brasileiros de Ginecologia, 27(5): 339-358, 1949.

TEIXEIRA, Luiz Antônio; LOWY, Ilana. "Imperfect Tools for a Difficult job: colposcopy, Colpocytology and Screening for Cervical Cancer in Brazil". In: Social Studies of Science, published online 13 June 2011.

SCHEFFEY, Lewis; BOLTEN, Karl; LANG, Warren. "Colposcopy: aid in diagnosis of cervical cancer”. In: Obstetrics and Gynecology. Vol. 5, number 3, March, 1955. 